EDITORIAL

\title{
What influences the outcome of valve replacement in critical aortic stenosis?
}

\section{H Baumgartner}

Heart 2005;91:1254-1256. doi: 10.1136/hrt.2004.055129

Even patients with severely reduced left ventricular function and critical aortic stenosis can improve notably following valve replacement

T he outcome of severe aortic stenosis (AS) is extremely poor once patients develop symptoms. $^{1{ }^{2}}$ In contrast, outcome becomes favourable when such patients undergo aortic valve replacement. ${ }^{34}$ Although results of available studies are not consistent in all aspects, preoperative severity of symptoms, presence of left ventricular (LV) dysfunction, and presence of coronary artery disease have been found to be major predictors of operative and long term mortality in most of them. ${ }^{4-8}$

\section{EARLY SURGERY AND THE OUTCOME OF VALVE REPLACEMENT}

Since severe symptoms and development of LV dysfunction are associated with a worse outcome following valve replacement, ${ }^{4-8}$ surgery should be performed early, before these characteristics occur. ${ }^{49}$ The question is: how early is early enough to guarantee an optimal long term outcome? There is general agreement that surgery should be performed immediately when symptoms occur even if they are only mild. ${ }^{9}$ In valvar heart disease in general, preservation of myocardial function has been identified as one of the major requirements to keep long term mortality and morbidity as low as possible. ${ }^{9}$ To achieve this goal valve replacement may, under certain circumstances, even be recommended in patients who are still asymptomatic. However, whereas development of irreversible left ventricular dysfunction is a major concern in asymptomatic patients with regurgitant lesions, ${ }^{9}$ this appears to be very uncommon in asymptomatic AS. Thus, observation of left ventricular size and function during the asymptomatic phase of severe AS is of minor importance. Nevertheless, there is agreement that the rare asymptomatic patient with already reduced LV dysfunction should undergo valve replacement (class IIa indication ${ }^{9}$ ).

There has been debate for many years over whether severe LV hypertrophy, which may be accompanied by myocardial fibrosis, increases operative risk and precludes an optimal long term outcome after valve replacement for AS. Although several retrospective studies reported the early and late prognostic importance of the extent of preoperatively increased LV mass, ${ }^{10}{ }^{11}$ there is insufficient evidence to justify elective surgery in asymptomatic patients just because LV mass has reached a certain cut-off. ${ }^{9}$ Since sudden death is very unlikely in truly asymptomatic patients, ${ }^{12}$ as is impaired LV function, current practice guidelines list the symptomatic patient with severe AS as the only class I indication for valve replacement. Unfortunately, patients frequently do not immediately present following occurrence of symptoms and some countries struggle with significant waiting times for heart surgery. This symptomatic yet unoperated phase of the disease puts patients at risk of sudden death and also at risk of further deterioration of their symptomatic state or LV function, which may again preclude an optimal outcome following valve replacement. Thus, risk stratification in asymptomatic patients is of critical importance. A positive stress test, ${ }^{13}$ particularly when symptoms are documented (abnormal blood pressure response and ST-T changes have less positive predictive value), and the presence of moderate or severe valve calcification together with an observed rapid haemodynamic progression (increase $>0.3 \mathrm{~m} / \mathrm{s}$ in peak transaortic velocity within one year), ${ }^{12}$ have been shown to identify high risk patients who are likely to develop problems in the near future and in whom elective surgery may be recommended (class IIa indications ${ }^{9}{ }^{14}$ ). We recently reported that plasma concentrations of neurohormones such as brain natriuretic peptide (BNP) and N-terminal (NT) BNP may predict symptom-free survival in AS as well as the outcome of valve replacement. ${ }^{15}$ Considering preoperative neurohormone concentrations, age, New York Heart Association (NYHA) class, aortic valve area, ejection fraction $(\mathrm{EF})$, and presence of coronary artery disease, we found that neurohormone values, EF, and NYHA class predicted survival; neurohormone values predicted postoperative symptomatic status; and neurohormone values and preoperative EF predicted postoperative EF by univariate analysis. By multivariate analysis, NT BNP was the only independent predictor of outcome. ${ }^{15}$ Thus, assessment of neurohormones may gain importance for determining timing of surgery in asymptomatic AS, although this requires further confirmation by larger studies.

\section{WHAT INFLUENCES OUTCOME OF LATE VALVE REPLACEMENT IN CRITICAL AORTIC STENOSIS WITH NOTABLY REDUCED LV FUNCTION?}

Unfortunately, we still encounter patients with critical AS who have been diagnosed late in an

Abbreviations: $A S$, aortic stenosis; $B N P$, brain natriuretic peptide; EF, ejection fraction; LV, left ventricular; NYHA, New York Heart Association 
advanced symptomatic state and who present with already notably reduced LV function. What is the outcome of valve replacement in these patients? So far, the largest experience in this patient group has been published by Connolly et $a l .{ }^{8}$ They reported the outcome of 154 patients with critical AS and preoperative $\mathrm{EF}<35 \%$. Additional bypass surgery was performed in $51 \%$. Perioperative mortality was $9 \%$. Although this mortality rate was higher than in the overall population with aortic valve replacement, it was nevertheless acceptable. Mean (SD) EF increased from $27(6) \%$ to 39 (14)\%. More importantly, symptomatic status notably improved with $88 \%$ of patients being in NYHA class III or IV before surgery compared to $7 \%$ postoperatively.

In this issue of Heart, Vaquette and colleagues ${ }^{16}$ confirm these results in a large patient cohort. In their retrospective analysis of 155 consecutive patients with severe AS and EF $<30 \%$, 30 day mortality was $12 \%$. EF increased even more, from $25(5) \%$ preoperatively to $47(12) \%$ at 12 months, and the percentage of patients in NYHA class III or IV decreased from $89 \%$ to $3 \%$. The major additional new finding of this study is that an early increase in EF $>10 \%$ predicts EF at one year and more importantly long term mortality. From a clinician's point of view, however, preoperative variables that predict outcome of valve replacement in this patient group would certainly be of greater interest. In the present study, the cardiothoracic ratio was the only independent predictor of operative mortality by multivariate analysis, although NYHA class, pulmonary artery pressure, and prevalence of complete left bundle branch block or renal insufficiency were significantly higher in non-survivors than in survivors. The prognostic relevance of LV size has also been reported by Tarantini and colleagues ${ }^{17}$ who identified $\mathrm{LV}$ end systolic volume index as the only independent predictor of cardiac death in their study of surgery in severe AS with reduced LV function. Diabetes, age $\geqslant 75$ years, and early EF change were the only independent predictors of long term mortality in the present study. The fact that preoperative EF did not predict outcome must certainly be viewed with caution. This certainly does not mean that LV function is not a predictor of outcome in AS in general, but this relation may no longer be obvious when looking at patients with notably reduced LV function only. In contrast to previous reports, presence of coronary artery disease ${ }^{6} 18$ and low transvalvar gradient ${ }^{78}$ were also not significant predictors of outcome in this study. This may, however, be explained by selection bias. The percentage of patients with coronary artery disease requiring bypass surgery was surprisingly low at $13 \%$, and only $11 \%$ of patients had a mean pressure gradient $\leqslant 30 \mathrm{~mm} \mathrm{Hg}$. The under representation of these characteristics may easily explain why the authors were not able to identify them as predictors of outcome. It is evident from numerous studies that aortic valve replacement with additional bypass surgery bears a significantly greater operative risk than aortic valve replacement without coronary surgery, and the presence of coronary artery disease has also been shown to worsen long term outcome. ${ }^{18}$ In the subset of patients with reduced LV function, the presence of coronary artery disease gains particular importance. In patients with irreversible ischaemic myocardial damage (scar), aortic valve replacement cannot be expected to improve wall motion in these segments. Less improvement in ventricular function may mean worse long term outcome as demonstrated in the present study. The fact that EF increased much more in the present study as compared with Connolly's report may indeed be due to the difference in the presence of coronary artery disease requiring bypass surgery $(51 \% \vee 13 \%)$.

\section{LOW FLOW-LOW GRADIENT AORTIC STENOSIS: THE MOST DIFFICULT PATIENT SUBSET}

Patients with critical AS, as indicated by small aortic valve area, who present with a low gradient at low cardiac output have been identified as a high risk subgroup in several previous studies. ${ }^{7}$ Gradients are well known to be flow dependent and therefore must decrease when LV function and transvalvar flow decreases. Nevertheless, the majority of patients with notably reduced LV function still present with mean gradients $>40 \mathrm{~mm} \mathrm{Hg.}{ }^{8}$ Patients with gradients $<30 \mathrm{~mm} \mathrm{Hg}$ have been reported to have a significantly higher operative mortality and worse long term outcome than those with higher gradients. ${ }^{7}$ Although the present study did not identify transvalvar gradient as a predictor of mortality, it was a predictor of early postoperative EF increase $>10 \%$ which in turn was a predictor of long term outcome. Thus, the data confirm at least indirectly the predictive value of a low gradient.

The concept has been raised that some of these patients with low gradients may not even have severe AS but only mild to moderate disease associated with other aetiologies of LV dysfunction such as cardiomyopathy or coronary artery disease. ${ }^{19}$ In this case, the opening forces may be too low to open the valve cusps to a greater extent. This has been called pseudosevere aortic stenosis. It has been suggested that dobutamine echocardiography may identify these patients. ${ }^{19}$ An increase in contractility and therefore transvalvar flow can separate patients in whom the valve area increases significantly with only a little increase in gradient (that is, pseudosevere stenosis) from those in whom the valve area remains small and the gradient increases significantly (that is, truly severe stenosis). Following this concept, patients with truly severe AS should be those who benefit most from valve surgery. Although reasonable, this concept still awaits confirmation in larger studies. A French multicentre study, ${ }^{20}$ the only published large scale study of patients with low flow-low gradient AS, was not able to comment on this concept, as pseudosevere AS by the definition above was very uncommon in their patient cohort. However, the investigators were able to demonstrate the prognostic value of contractile reserve. Patients with an increase in stroke volume $\geqslant 20 \%$ had the best outcome after valve replacement whereas outcome was very poor when these patients were followed medically. Patients without contractile reserve had a poor outcome which did not differ significantly between patients who underwent surgery and those who did not. Heart transplantation may have to be considered in these patients.

In conclusion, the study by Vaquette and colleagues ${ }^{16}$ once more confirms the message that even patients with severely reduced LV function and critical AS can improve notably with valve replacement. However, the difficult question as to whether there are patients in this subset of the AS population who should undergo conservative treatment and eventually heart transplantation because they have pseudosevere rather than truly severe stenosis still remains to be answered.

\section{REFERENCES}

1 Ross J Jr, Braunwald E. Aortic stenosis. Circulation 1968;38(1 suppl):61-7.

2 Horstkotte D, Loogen F. The natural history of aortic valve stenosis. Eur Heart J 1988;9(suppl E):57-64

3 Schwarz F, Baumann P, Manthey J, et al. The effect of aortic valve replacement on survival. Circulation 1982;66:1 105-10.

4 Lund $\mathrm{O}$. Preoperative risk evaluation and stratification of long-term survival after valve replacement for aortic stenosis. Reasons for earlier operative intervention. Circulation 1990;82:124-39.

5 Kvidal P, Bergstrom R, Horte LG, et al. Observed and relative survival after aortic valve replacement. J Am Coll Cardiol 2000;35:747-56.

6 Lund O, Flo C, Jensen FT, et al. Left ventricular systolic and diastolic function in aortic stenosis. Prognostic value after valve replacement and underlying mechanisms. Eur Heart J 1997;18:1977-87. 
7 Connolly HM, Oh JK, Schaff HV, et al. Severe aortic stenosis with low transvalvular gradient and severe left ventricular dysfunction: result of aortic valve replacement in 52 patients. Circulation 2000;101:1940-6.

8 Connolly HM, Oh JK, Orszulak TA, et al. Aortic valve replacement for aortic stenosis with severe left ventricular dysfunction. Prognostic indicators. Circulation 1997;95:2395-400.

9 Bonow RO, Carabello B, de Leon AC, et al. ACC/AHA guidelines for the management of patients with valvular heart disease. A report of the American College of Cardiology/American Heart Association task force on practice guidelines (committee on management of patients with valvular heart disease) J Am Coll Cardiol 1998;32:1486-588.

10 Mehta RH, Bruckman D, Das S, et al. Implications of increased left ventricular mass index on in-hospital outcomes in patients undergoing aortic valve surgery. J Thorac Cardiovasc Surg 2001;122:919-28.

11 Orsinelli DA, Aurigemma GP, Battista S, et al. Left ventricular hypertrophy and mortality after aortic valve replacement for aortic stenosis: a high risk subgroup identified by preoperative relative wall thickness. J Am Coll Cardiol 1993;22:1679-83.

12 Rosenhek R, Binder T, Porenta G, et al. Predictors of outcome in severe, asymptomatic aortic stenosis. N Engl J Med 2000;343:611-7.

13 Amato MC, Moffa PJ, Werner KE, et al. Treatment decision in asymptomatic aortic valve stenosis: role of exercise testing. Heart 2001;86:381-6.

14 lung B, Gohlke-Barwolf, Tornos P, et al. on behalf of the Working Group on Valvular Heart Disease Recommendations on the management of the asymptomatic patient with valvuar heart disease. Eur Heart $\rfloor$ 2002;23:1253-66

15 Bergler-Klein J, Klaar U, Herger M, et al. Natriuretic peptides predict symptom-free survival and postoperative outcome in severe aortic stenosis. Circulation 2004; 109:2302-8.

16 Vaquette B, Corbineau $H$, Laurent $M$, et al. Valve replacement in patients with critical aortic stenosis and depressed left ventricular function: predictors of operative risk, left ventricular function recovery, and long term outcome. Heart 2005;91:1324-9.

17 Tarantini G, Buja P, Scognamiglio R, et al. Aortic valve replacement in severe aortic stenosis with left ventricular dysfunction: determinants of cardiac mortality and ventricular function recovery. Eur J Cardiothorac Surg 2003;24:879-85

18 Smith WT, Ferguson TB, Ryan T, et al. Should coronary artery bypass graft surgery patients with mild or moderate aortic stenosis undergo concomitant aortic valve replacement? A decision analysis approach to the surgical dilemma. J Am Coll Cardiol 2004;44:1241-7.

19 defilippi CR, Willett DL, Brickner ME, et al. Usefulness of dobutamine echocardiography in distinguishing severe from nonsevere valvular aortic stenosis in patients with depressed left ventricular function and low transvalvular gradients. Am J Cardiol 1995;75:191-4.

20 Monin JL, Quéré JP, Monchi M, et al. Low-gradient aortic stenosis operative risk stratification and predictors for long-term outcome: a multicenter study using dobutamine strss hemodynamics. Circulation 2003;108:319-24.

\section{IMAGES IN CARDIOLOGY}

\section{Armoured heart}

A 49 year old man with a history of tuberculosis during childhood was referred to our department for a routine check up. The chest radiograph (left panel) showed circumferential calcification of the heart (arrows). Subsequently performed ECG gated multislice computed tomography (right panel) confirmed pericardial calcifications (arrows) surrounding nearly the entire heart without involving the coronary arteries (asterisk). The left ventricular ejection fraction was estimated to be normal by computed tomography and transthoracic echocardiography (to view video footage visit the Heart websitehttp://www.heartjnl.com/supplemental). Different causes of pericardial calcifications have to be considered: tuberculosis, radiation therapy, trauma, uraemia, and viral infections. In the patient presented here the episode of tuberculosis during childhood is the most likely cause of the armoured heart.
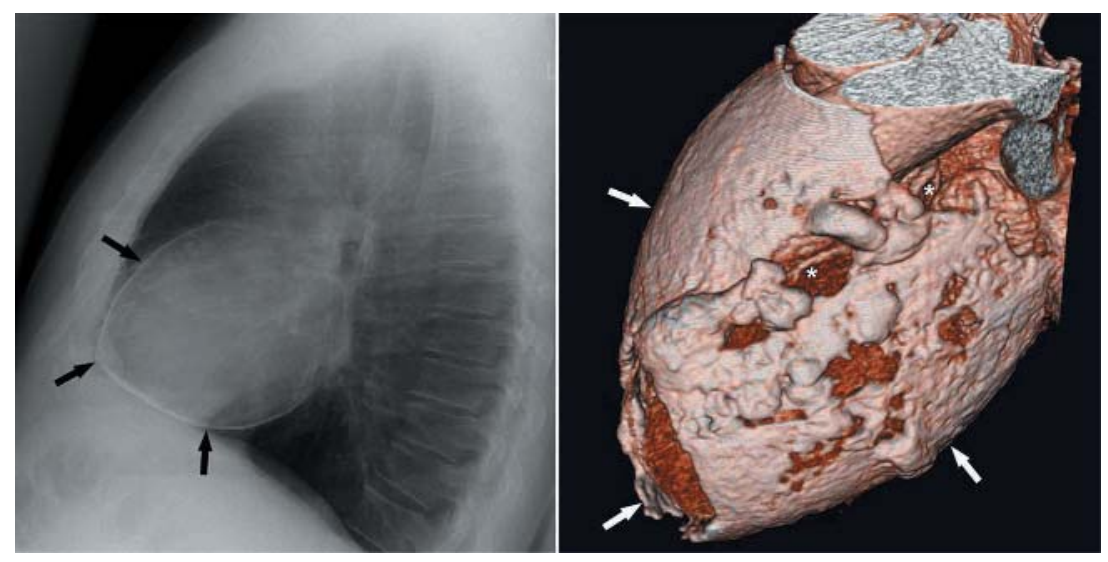

M Dewey

S Eddicks

B Hamm marc.dewey@charite.de

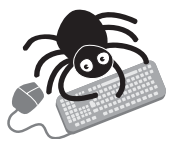

To access video files visit the Heart websitehttp://www.heartjnl.com/supplemental 